\title{
New species of Homidia (Collembola, Entomobryidae) from eastern China with description of the first instar larvae
}

\author{
Zhi-xiang Pan ${ }^{1, \dagger}$, Shi-di Shi ${ }^{1, \ddagger}$, Feng Zhang ${ }^{2, \S}$ \\ I School of Life Sciences, Taizhou University, Linhai, Zhejiang 317000, China 2 School of Life Sciences, \\ Nanjing University, Nanjing, Jiangsu 210093, China \\ † urn:lsid:zoobank.org:author:0BF369B1-05D5-4141-9D03-A2135DF1AF94 \\ ¥ urn:lsid:zoobank.org:author:01C93AAA-7FF4-47E9-A024-17DEC7A116EE \\ § urn:lsid:zoobank.org:author:904C4445-AAEA-40BC-B418-502053EF39B8 \\ Corresponding author: Feng Zhang (xtmtd.zf@gmail.com)
}

Academic editor: L. Deharveng | Received 30 April 2011 | Accepted 3 November 2011 | Published 8 December 2011

urn:lsid:zoobank.org:pub:DE876BFB-D04D-4C87-B972-83EBD12C8ACB

Citation: Pan Z-x, Shi S-d, Zhang F (2011) New species of Homidia (Collembola, Entomobryidae) from eastern China with description of the first instar larvae. ZooKeys 152: 21-42. doi: 10.3897/zookeys.152.1455

\begin{abstract}
Morphology of the first instar larvae of Collembola has considerably taxonomical and phylogenetic significance. We describe the first instar larvae for the first time in Homidia. External morphology of first instar larvae and adults of Homidia jordanai sp. $\mathrm{n}$. is described based on observations under light and scanning electron microscopes. Most organs of adults bear considerably more setae than the first instar larvae; in addition, first instar larval Homidia lack labial seta $\mathrm{R}$, seta on tenaculum, mucronal spine, and dental spines. The new species is characterized by weakly pigmented body, long antennae subequal to body in length, $1+1$ inner macrochaetae on Abd. III, few inner macrochaetae on posterior Abd. IV, and spiny and short seta pi on dental base. Differences between new species and other two similar ones, taxonomical significance of the first instar larvae and the position of Homidia are also discussed.
\end{abstract}

Copyright Zx. Pan, Sd. Shi, F. Zhang. This is an open access article distributed under the terms of the Creative Commons Attribution License, which permits unrestricted use, distribution, and reproduction in any medium, provided the original author and source are credited. 


\section{Keywords}

new species, first instar, scanning electron microscope, chaetotaxy, China

\section{Introduction}

In epimetabolic Collembola, the number of setae and pigmentation often change after moult (Christiansen 1992). Morphology of the first instar larvae has considerably taxonomical and phylogenetic significance (Szeptycki 1979), but it has been rarely studied. Homidia is the most widespread entomobryid genus in East Asia, particularly Japan, Korea and China. The genus is characterized by presence of spines on inner edge of dens, "eyebrow" macrochaetae on anterior part of Abd. IV of adults, ommatidia $8+8$, mucro bidentate with subapical tooth much larger than the apical one, and absence of body scales. The chaetotaxy of first instar larvae (primary chaetotaxy) in Homidia has never been described.

In the present paper, the primary chaetotaxy of Homidia was studied for the first time based on $H$. jordanai sp. n., from East China. Many other morphological details are showed under scanning electron microscope.

\section{Material and methods}

Alcohol preserved young and adult specimens, were cleared in lactic acid, mounted on microscope slides in Marc André II solution, and studied using Leica DM2500 and Nikon 80i microscopes. Few specimens, coated with platimum under vacuum conditions, were observed under scanning electron microscope. Photographs were taken with Leica AL2 and Nikon SMZ1000 microscopes using a mounted Nikon DS-Fil camera and Hitachi $\$ 4800$ scanning electron microscope, numbers and letters added with photoshop CS2 (Adobe Inc.), all length data measured with Nikon NIS-Elements Documentation 3.1. First instar larvae were determined by the stability of primary chaetotaxy (e.g. 19/18 common setae on meso-/metathorax, Szeptycki 1979). Cephalic dorsal chaetotaxy is designated after Jordana and Baquero (2005) and Soto-Adames (2008), interocular setae after Mari-Mutt (1979, 1986), labial palp setae after Fjellberg (1998), labial setae after Gisin (1964), dorsal chaetotaxy after Szeptycki (1979).

Abbreviations. Th. -thoracic segment, Abd. -abdominal segment, Ant. -antennal segment, ms -microsensillum/a, s-common sensillum/a, mac-macrochaeta(e), mic -microchaeta(e), SEM -scanning electron microscope, LM -light microscope, Tita IIII -tibiotarsus of fore, mid, and hind legs. 


\section{Results}

\section{Homidia jordanai sp. n.}

urn:lsid:zoobank.org:act:7A40BC6A-99BB-4A02-AE92-0899F6556BD8

http://species-id.net/wiki/Homidia_jordanai

Figs 1-80, Tables 1-3

Holotype. $\widehat{\delta}$ on slide, Shaoxin City, Zhuji Country, Dongbaihu, Zhejiang Province, CHINA, $29^{\circ} 34.48^{\prime} \mathrm{N}, 120^{\circ} 24.32^{\prime} \mathrm{E}, 3 . X .2009$, collection number $\$ 4014$, collected by Zhi-Xiang Pan and Chen-Chong Si, deposited in Taizhou University.

Paratypes. 20,11 and 5 larvae on slide, numerous in alcohol, same data as holotype. 5 paratypes ( $1 \hat{\sigma}, 1 \%$ on slide, 1 larva and 2 adults in alcohol) deposited in School of Life Sciences, Nanjing University and others in Taizhou University, China.

Etymology. Named after the famous Spanish entomologist Jordana Rafael (University of Navarra).

Description. Adult. Size. Maximum body length up to $2.3 \mathrm{~mm}$.

Habitus. Ground colour pale yellow in alcohol. Body dorsally without pigment. Coxa and trochanter of all legs with weak blue pigment. Eye patches dark. Antennae gradually darker from Ant. III to Ant. IV (Figs 1, 2).

Head. Ommatidia 8+8, $\mathrm{G}$ and $\mathrm{H}$ smaller than others, and sometimes invisible under LM, interocular setae as p, r, t (Figs 5, 44). Antenna 3.9-4.6 times as long as cephalic diagonal, subequal to body in length, antennal segment ratio as I : II : III : IV $=1: 1.3-1.5: 1.3-1.4: 2.4-3.1$. Basal Ant. I with 2 dorsal and 4 ventral spiny setae (Figs 5, 45, 46). Basal Ant. II with 5 smooth setae (Figs 47, 48); distal Ant. II with 4 s (2-3 longer, 1-2 shorter) (Figs 52, 53). Ant. III organ with 2 rod-like $s$ and 3 small $s$ (Figs 54-56); those $s$ also with obvious ridges on surface under SEM. Distal Ant. IV with several types of s (Figs 57-62); apical bulb bilobed (Fig. 6). Dorsal cephalic chaetotaxy with $4 \mathrm{An}$ and $7 \mathrm{~S}$ mac. Clypeus with many ciliate setae (Fig. 5). Labral papillae absent. Prelabral and labral setae as 4/5, 5, 4, all smooth, labium intrusion U-shaped (Fig. 7). Maxillary outer lobe with 1 apical seta, 1 subapical seta and 3 sublobal hairs on sublobal plate; subapical seta slightly longer than apical one (Fig. 49). Labial palp with five papillae A-E, with 0, 5, 0, 4, 4 guard setae, respectively, and 5 smooth proximal smooth setae; lateral process differentiated with blunt tip reaching apex of papilla E (Figs 8, 50, 51). Hyaline plate with 1 main $(H)$ and 2 accessorial $\left(h_{1}, h_{2}\right)$ setae. Setal formula of labial base as $\mathrm{MREL}_{1} \mathrm{~L}_{2}$, seta $\mathrm{E}$ smooth, others ciliate (Fig. 9).

Thorax. Complete s-chaetae of dorsal body as 32/223(>47)3 (examined specimens mostly with $47 \mathrm{~s}$-chaetae on Abd. IV, but some lost during preparation), ms as 10/10100. Th. II with 3 medio-medial (m1, m2, m2i), 3 medio-sublateral (m4, m4i, $\mathrm{m} 4 \mathrm{p}), 17-18$ posterior mac and $3 \mathrm{~s}$-chaetae ( $\mathrm{ms}$ antero-internal to s); seta p1i2 rarely present, seta $\mathrm{p} 6$ as mic. Th. III with 29-32 mac and 2 s-chaetae; setae p1i2 and p4 absent (Fig. 10). Numerous setae on hind leg (Figs 15-19); pseudopores on coxa shown in Fig. 63, but their number unclearly seen. Coxal macrochaetal formula as 3/4+1, $2+1 / 4+2$ (Fig. 11). Trochanteral organ with 36-64 smooth spiny setae (Fig. 16). Inner 

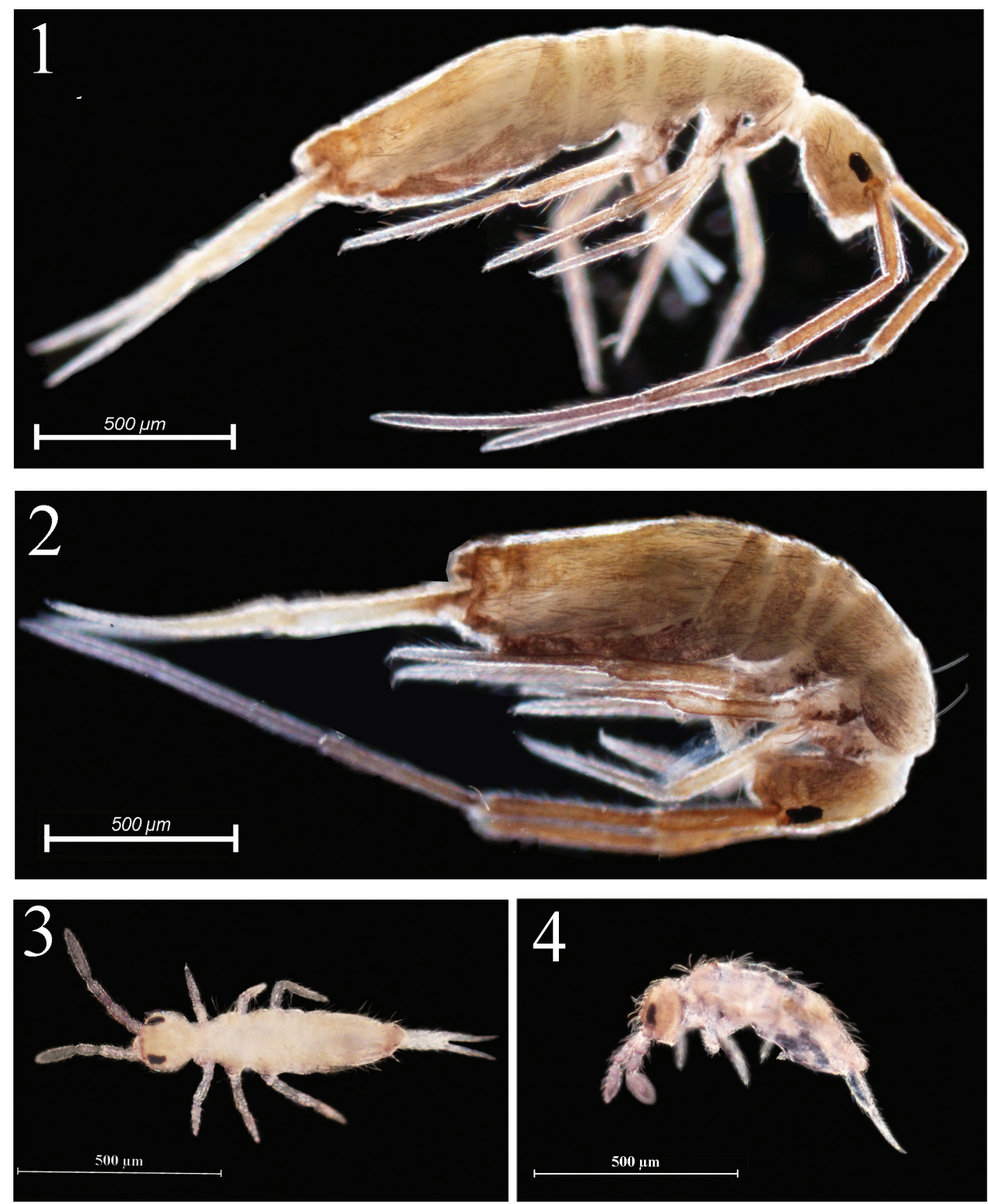

Figures I-4. Habitus of $H$. jordanai sp. n. I-2 adults, lateral view 3-4 the first instar larvae $\mathbf{3}$ dorsal view 4 lateral view.

differentiated tibiotarsal setae slightly ciliate, most distal smooth seta present on hind leg (Figs 18, 19, 65). Tenent hair clavate and subequal to inner edge of unguis in length (Figs 19, 66). Unguis with 4 inner, 2 lateral and 1 outer teeth, all tiny. Unguiculus lanceolate with outer edge slightly serrate (Fig. 65). Pretarsus with 1 pair of small spines (Figs 19, 67). 


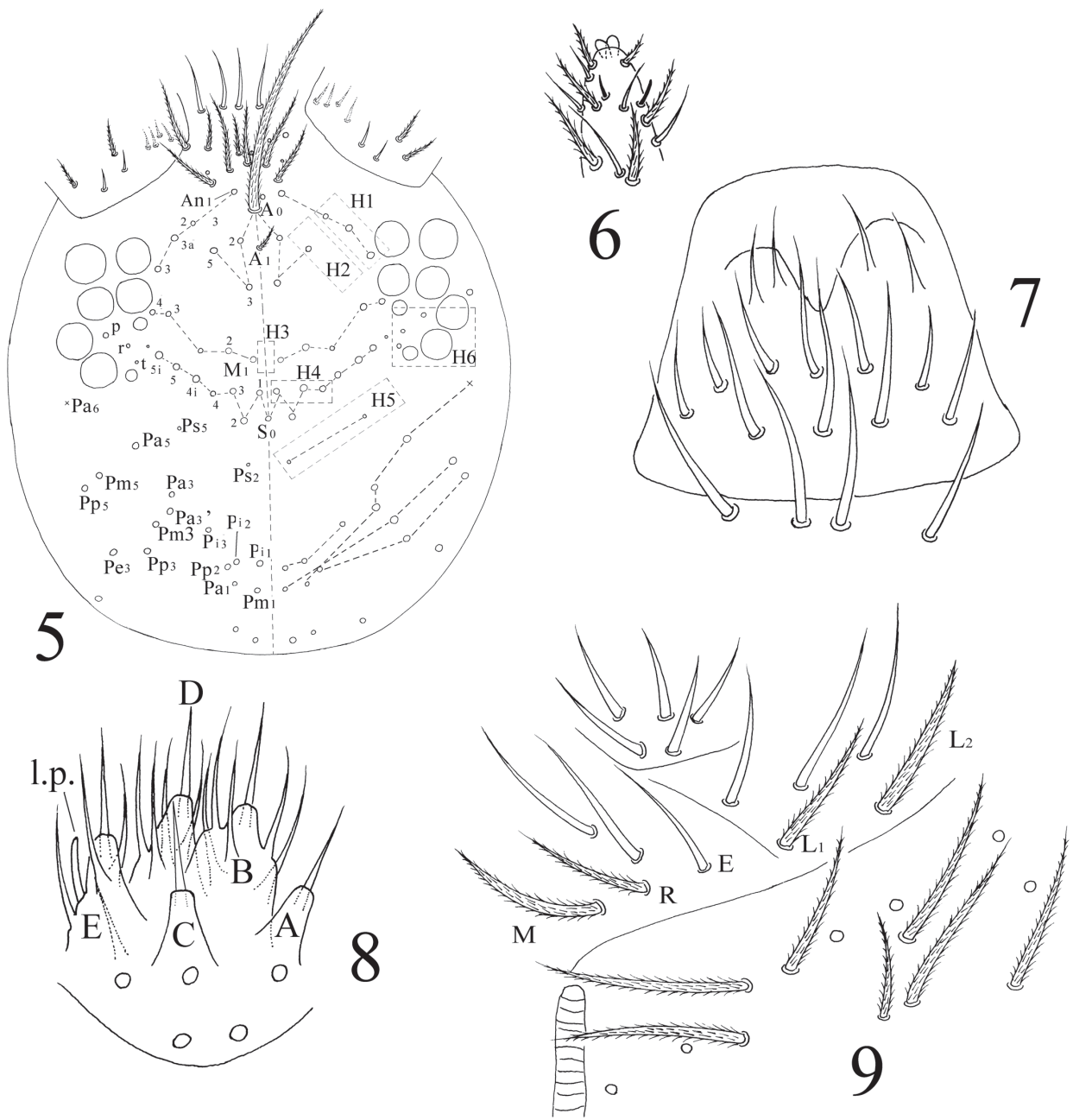

Figures 5-9. H. jordanai sp. n. 5 dorsal cephalic chaetotaxy $\mathbf{6}$ apical bulb of Ant. IV 7 labrum 8 labial palp 9 labial base.

Abdomen. Abd. I with 9 (a2, a3, m2-4, m2i, m4i, m4p, a5) mac and 2 s-chaetae (ms anterio-external to s) (Figs 68, 69). Abd. II with 6 (a2, a3, m3, m3e, m3ea, m3ep) inner, 1 (m5) lateral mac, and 2 s-chaetae. Abd. III with 1 (m3) inner and 4 (am6, pm6, m7a, p6) lateral mac, and 3 s-chaetae (Fig. 12). Abd. IV with more than 47 s-chaetae ( 2 of normal length and others elongated), 6-9 mac on anterior part and irregularly arranged in a transverse row; posterior part with 2 (3) (B5 and A6, A6 rarely as mic, B4 sometimes present) mac and 1 (B6) mic (Fig. 13). Abd. V with 3 s-chaetae; $\mathrm{m} 3 \mathrm{a}$ absent, a5i sometimes absent (Fig. 14). Anterior face of ventral tube with many ciliate setae, including $4+4 \mathrm{mac}$, line connecting proximal $(\mathrm{Pr})$ and external-distal $(\mathrm{Ed})$ (Chnd and $\mathrm{Li}$ 1997) mac oblique to median furrow (Fig. 20); posterior face with 5 or 6 (median with 1 or 2 small) smooth and numerous ciliate setae (Fig. 21); lateral 

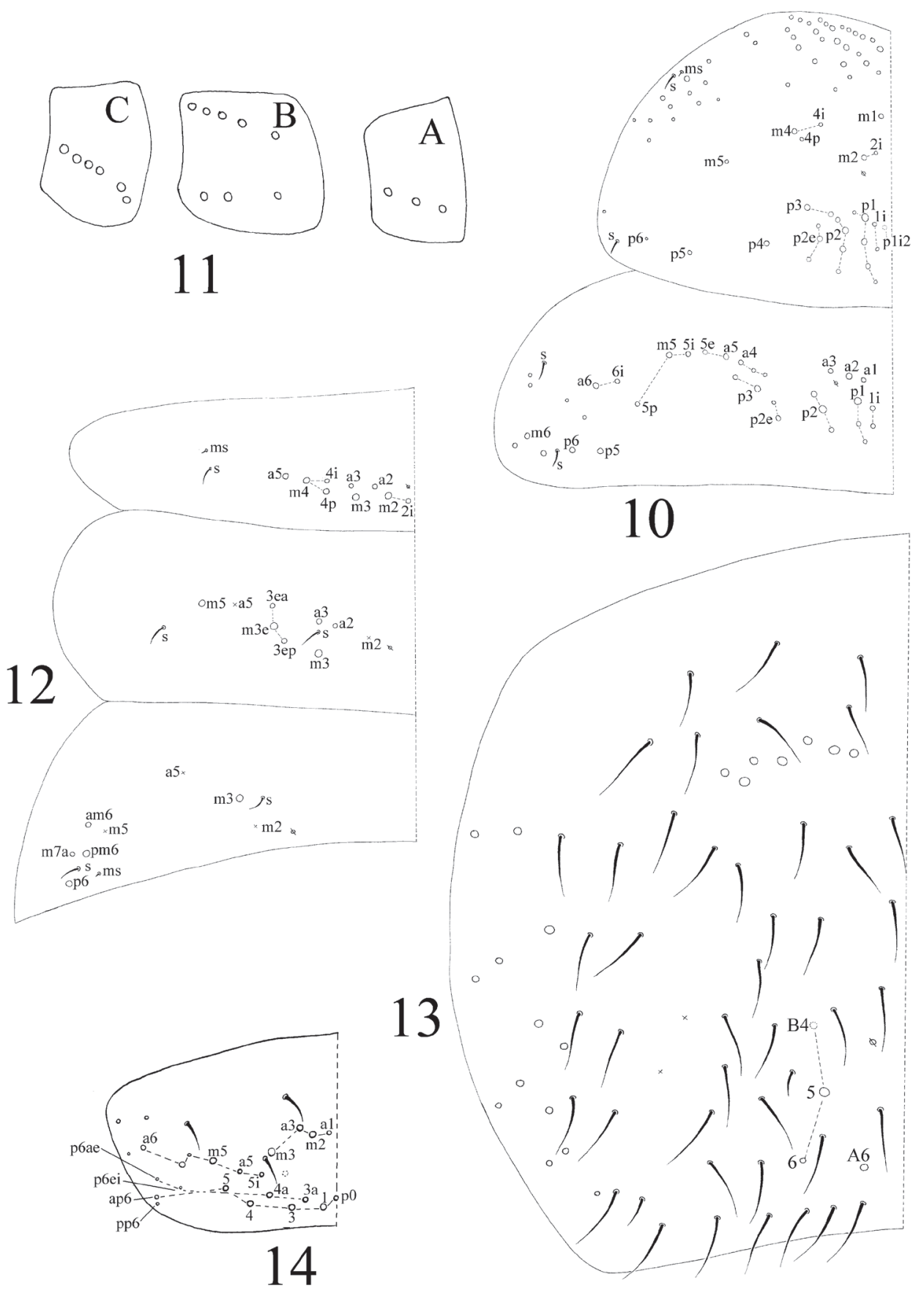

Figures 10-14. H. jordanai sp. n. I 0 dorsal chaetotaxy of Th. II-III I I coxal chaetotaxy formula (A fore leg $\mathbf{B}$ mid leg $\mathbf{C}$ hind leg) I 2-I $\mathbf{4}$ dorsal chaetotax I 2 Abd. I-III I 3 Abd. IV I4 Abd. V. 


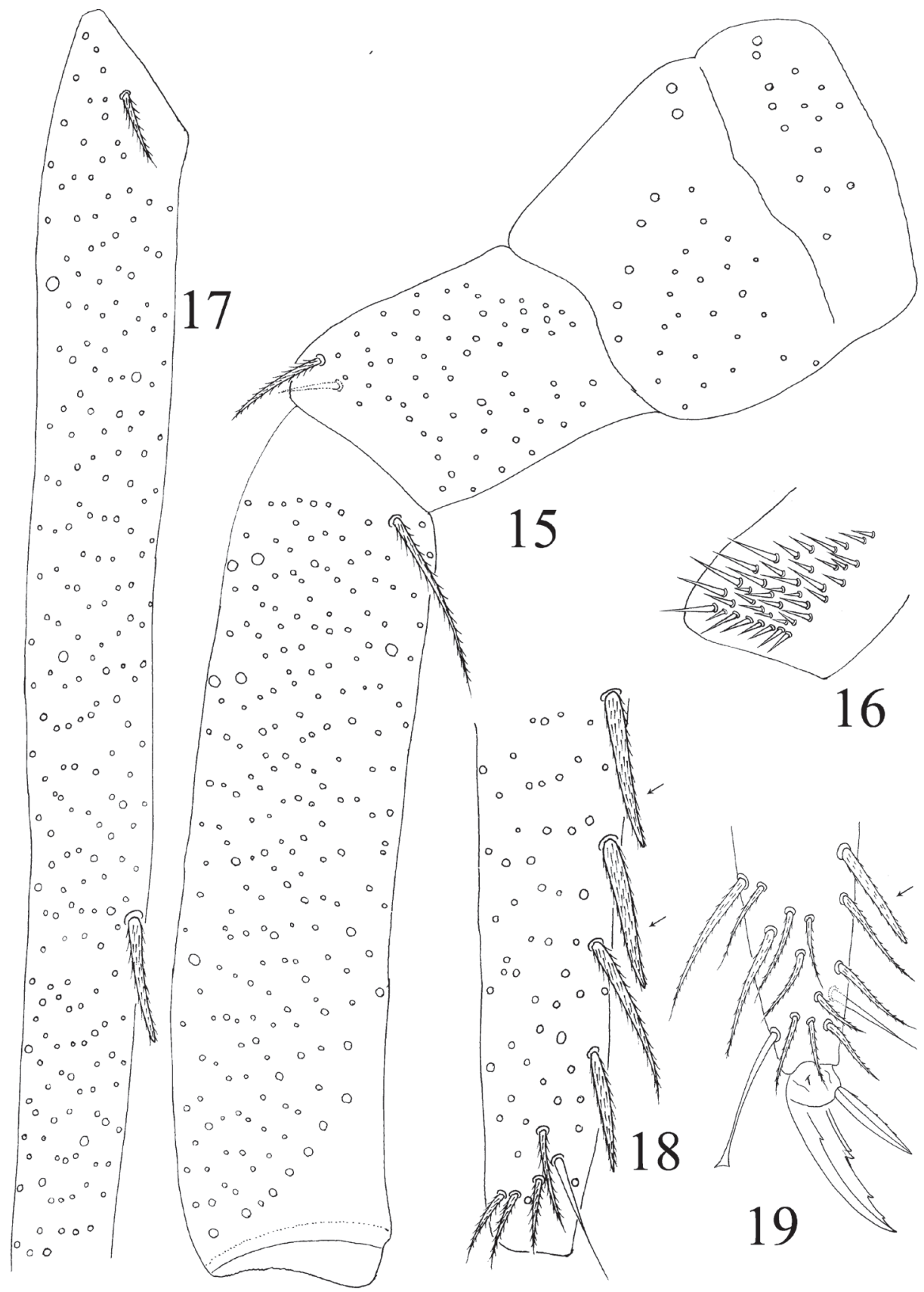

Figures 15-19. Hind leg of $H$. jordanai sp. n. $\mathbf{1 5}$ coxa, trochanter and femur $\mathbf{1 6}$ trochanteral organ $\mathbf{1 7}$ basal Tita 18 apical Tita 19 apical Tita and claw. 

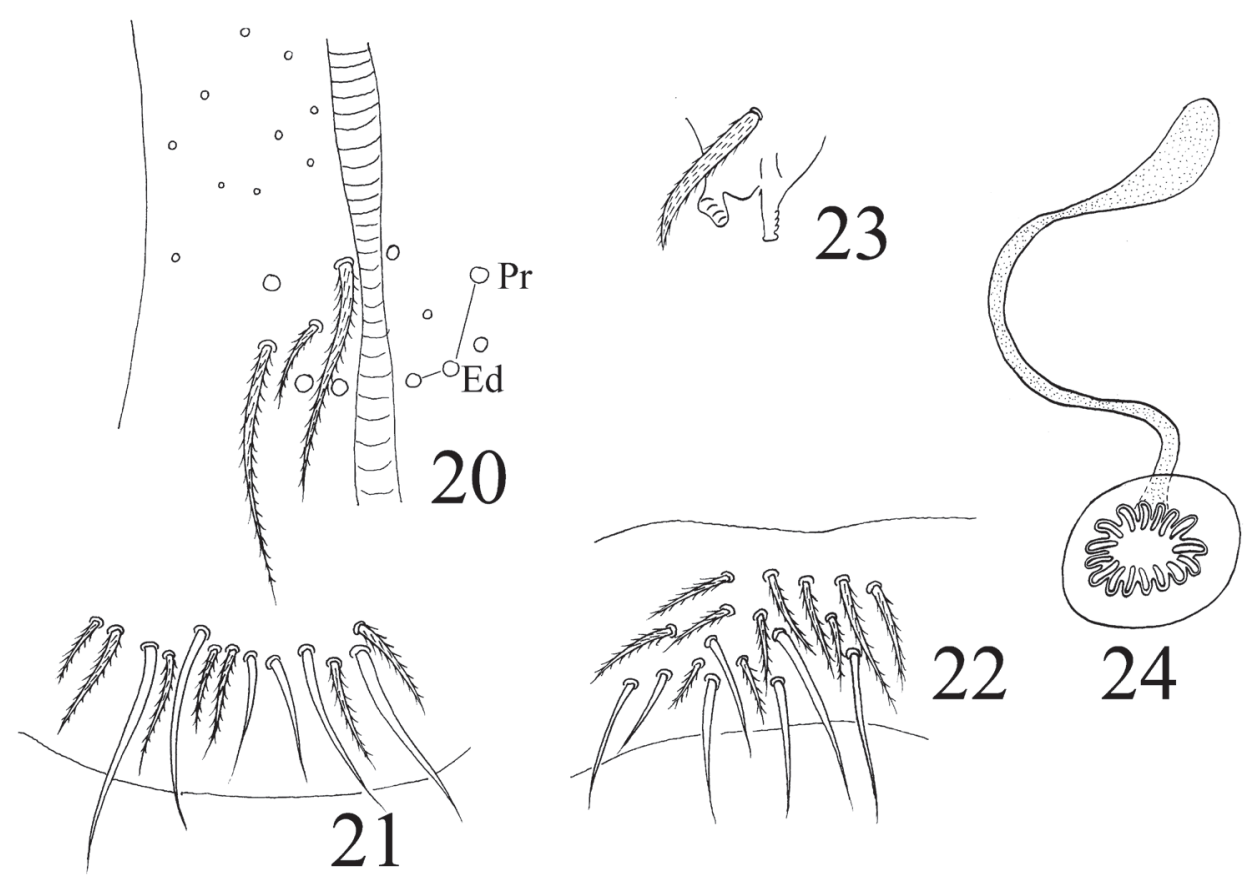

Figures 20-24. $H$. jordanai sp. n. 20-22 ventral tube $\mathbf{2 0}$ anterior face $\mathbf{2} \mathbf{I}$ posterior face $\mathbf{2 2}$ lateral flap 23 tenaculum 24 genital plate and spermary.

flap with 6-7 smooth and 10-22 ciliate setae (Figs 22, 74, 75). Furcula shown in Figs 25-28. Manubrial plaque with 3 pseudopores, 2 inner and 5-6 outer ciliate setae (Fig. 26). Dens with 20-40 spines (Figs 26, 27, 77); basal sete (Szeptycki 1973) bs ${ }_{1}$ and bs spiny and multilaterally ciliate, bs shorter than $\mathrm{bs}_{2}$; proximal-inner seta (pi) spiny, shorter and thicker than $\mathrm{bs}_{1}$ and $\mathrm{bs}_{2}$ (Figs 26, 27). Mucro bidentate with subapical tooth obviously larger than apical one; basal spine short, with tip only reaching apex of subapical tooth (Figs 28, 76). Tenaculum with $4+4$ teeth and 1 large, multilaterally ciliate basal seta (Fig. 23). Genital plate papillate (Fig. 24).

The first instar larva. Size. Body length up to $0.7 \mathrm{~mm}$.

Habitus. Ground colour pale white in alcohol. Eye patches dark. Distal antennae slightly pigmented (Figs 3, 4).

Head. Antenna 1.3-1.8 times as long as cephalic diagonal, antennal segment ratio as I : II : III : IV = $1: 1.4-2.2: 1.5-2.5: 3.3-4.3$. Ant. I with 11 ciliate and 1 basal spiny setae. Ant. II with 25 ciliate setae. Ant. III with 38 ciliate setae and 5 s-chaetae of Ant. III organ (Figs 30, 78). Ant. IV with numerous ciliate setae and some s-chaetae (Figs 31, 79). Dorsal cephalic chaetotaxy as $3 A n$ and $5 S$ mac (Fig. 29). Labium with 3 smooth proximal setae. Setal formula of labial base as $\mathrm{MEL}_{1} \mathrm{~L}_{2}$, seta $\mathrm{E}$ smooth, all others ciliate (Fig. 32). Ommatidia, Ant. IV apical bulb, interocular setae, labral papillae, labrum, maxillary outer lobe, labial palp, hyaline plate same as adults.

Chaetotaxy. Complete s-chaetae of body as 32/223(50-53)3, ms as 10/10100. Th. II with 13 (a1-6, m1, m4, m6, p1-3, p5) mac, 6 (a7, m2, m5, m7, p4, p6) mic and 


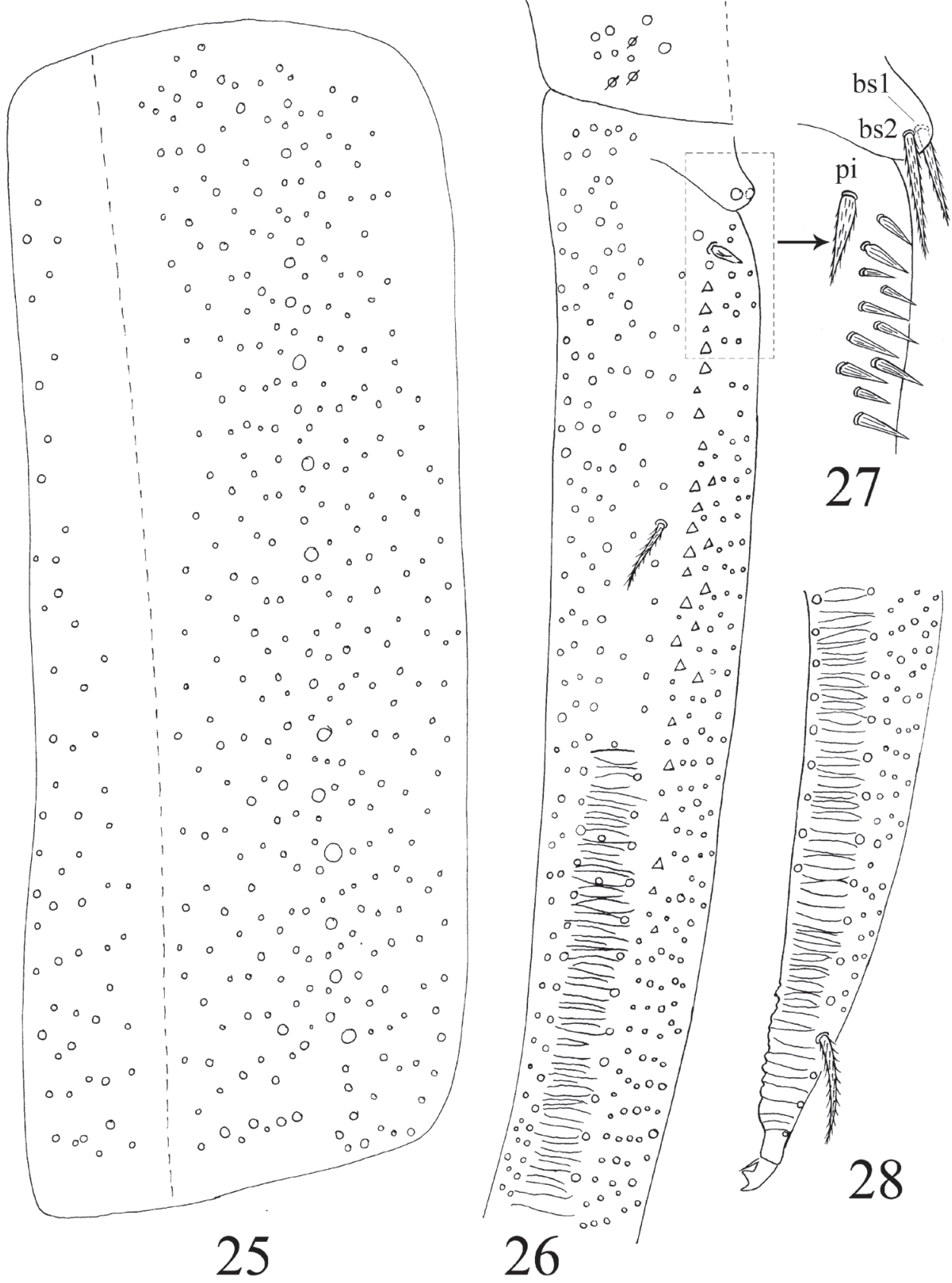

Figures 25-28. Furcula of $H$. jordanai sp. n. 25 manubrium 26 manubrial plaque and base of dens $\mathbf{2 7}$ inner side of dens (triangles representing spines) $\mathbf{2 8}$ apical dens and mucro. 

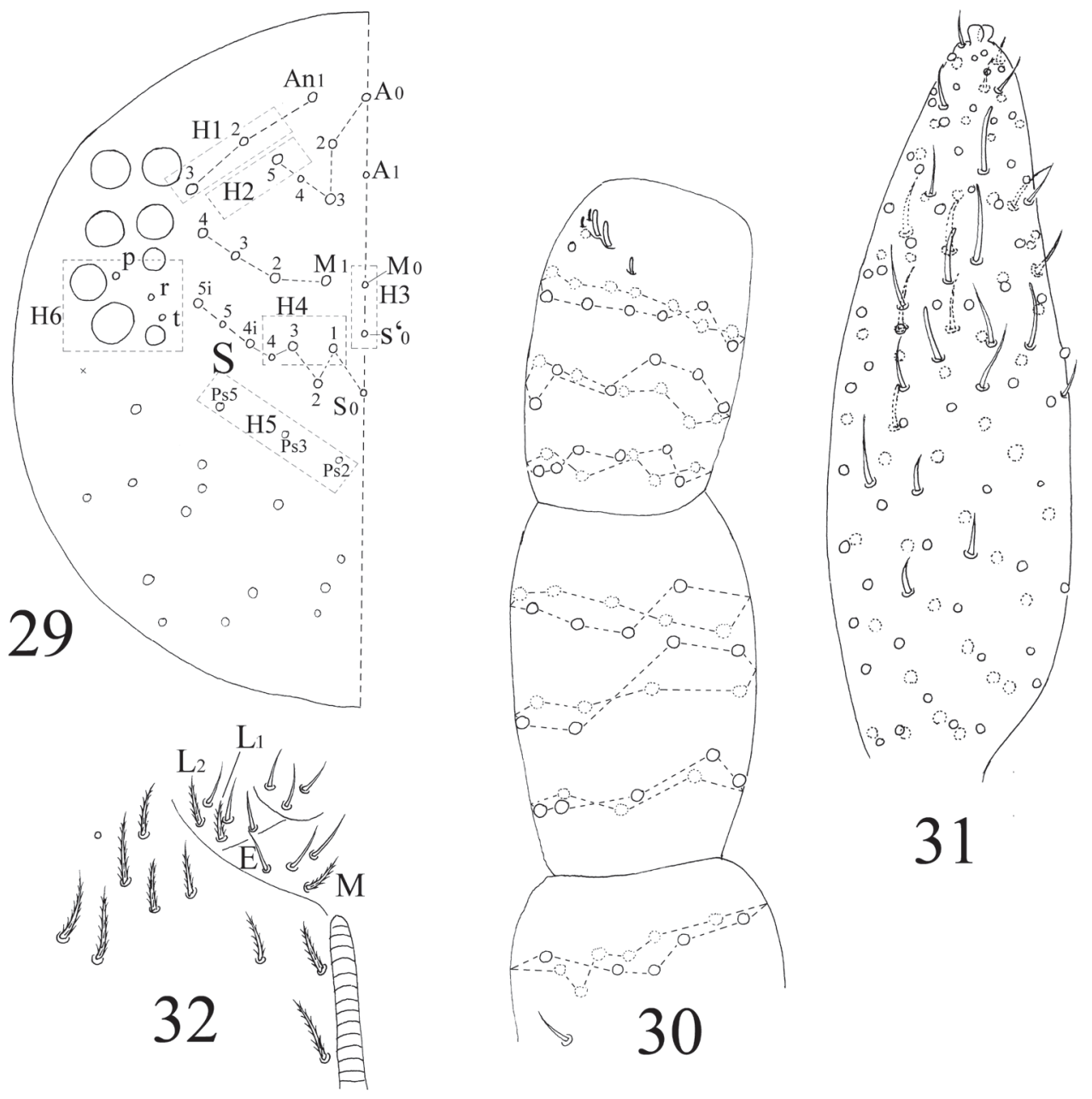

Figures 29-32. The first instar larval $H$. jordanai sp. n. 29 dorsal cephalic chaetotaxy $\mathbf{3 0}$ Ant. I-III 31 Ant. IV 32 labal base

3 s-chaetae (ms anterior to s), $\mathrm{m} 1$ rarely as mic. Th. III with 9 (a2-6, m6, p1-3) mac, 9 (a1, a7, m1, m4, m5, m7, p4, p6) mic and 2 s-chaetae. Abd. I with 3 (m2-4) mac, 9 (a1-3, a5, a6, m5, m6, p5, p6) mic and 2 s-chaetae (ms antero-external to s). Abd. II with $2(\mathrm{~m} 3, \mathrm{~m} 5)$ mac, 13 (a1-3, a6, a7, m4, m6, m7, p4, p7, el) mic, 1 additional mic on lateral and 2 s-chaetae. Abd. III with 1 (m3) mac central, 13 (a1-3, a6, a7, m4, am6, pm6, m7, p4) mic, 3 s-chaetae and 5 lateral additional mic (Fig. 33). Abd. IV with 2 (B5, E3) mac, 27 (A1-4, A6, B1-4, B6, C1-4, T1, T3, T5, D1-3, E1, E4, F1-3) mic and 50-53 s-chaetae (48-51 elongated and 2 of normal length); setae A5 and E2 absent (Fig. 34). Abd. V with total 14 setae and 3 s-chaetae. Abd. VI with $21+21$ setae; 3 on middle line (Fig. 35 ). 

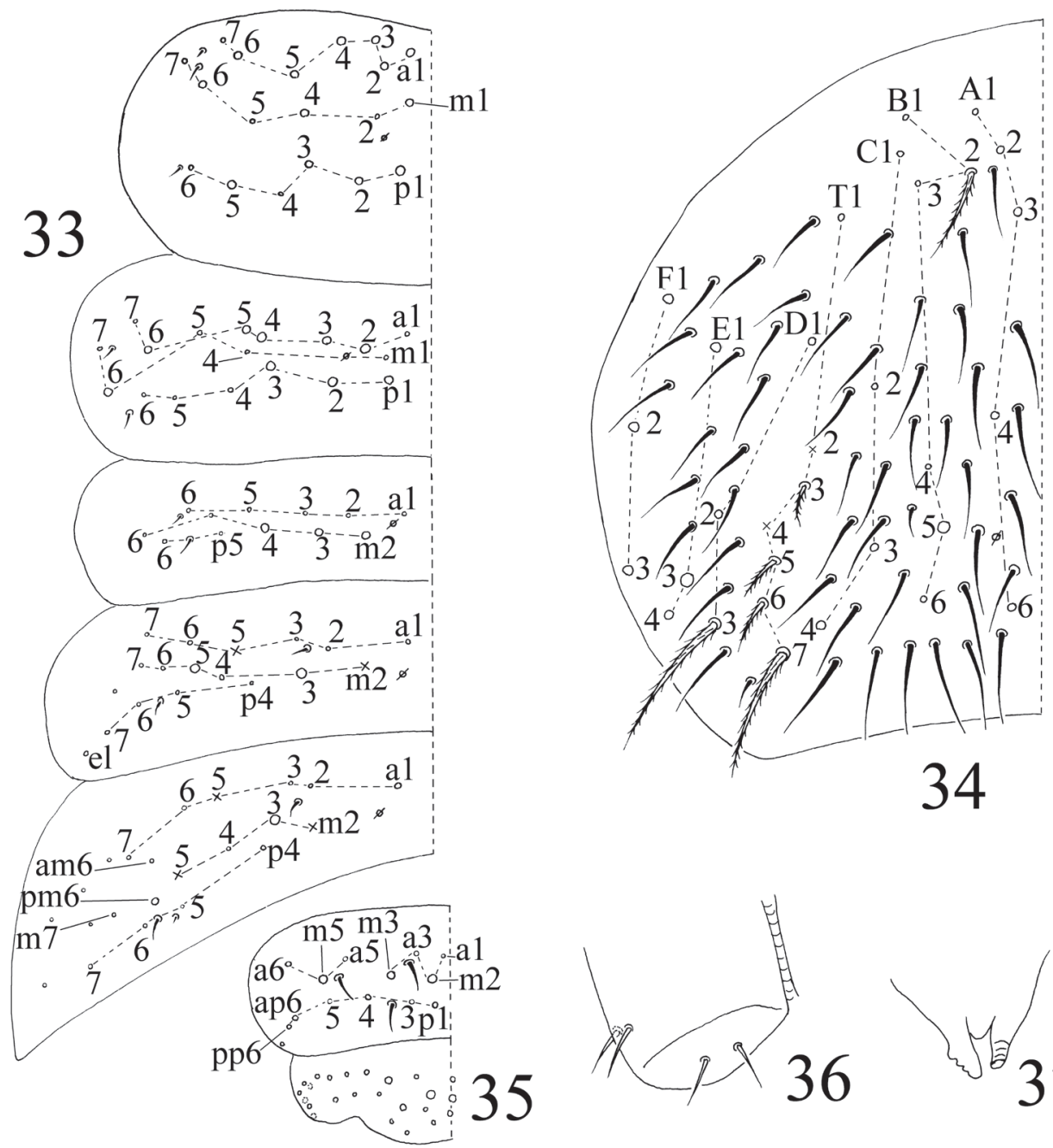

Figures 33-37. The first instar larval $H$. jordanai sp. n. 33-35 dorsal chaetotaxy 33 Th. II-Abd. III 34 Abd. IV 35 Abd. V-VI 36 ventral tube $\mathbf{3 7}$ tenaculum.

Leg. Coxa I-III with 2, 3, 5 ciliate setae. Trochanter I-III with 4, 5, 4 ciliate and 2, 1, 0 smooth setae, 1 spine on trochanter III. Femur I-III with 10, 16, 14 ciliate and 3, 1,3 smooth setae. Tita I-III with 38, 41, 46 ciliate setae and 1 tenent hair respectively, 1 supraempodial seta on Tita III (Figs 38-41). Unguis with 4 minute inner and 2 lateral teeth. Unguiculus lanceolate with outer edge slightly serrate (Fig. 80).

Ventral tube. Anterior face without seta; posterior face with 2 apical smooth setae; lateral flap with 2 smooth setae (Fig. 36).

Furcula. Manubrum with $24+24$ ciliate setae. Manubrial plaque not seen. Dorsal dens with 14-15 (8 in outer 6-7 in inner row) setae, ventral side with about 55 ciliate setae, inner of dens without dental spine, basal setae $\left(\mathrm{bs}_{1}\right.$ and $\mathrm{bs}_{2}$ ) absent (Figs 42, 43). 


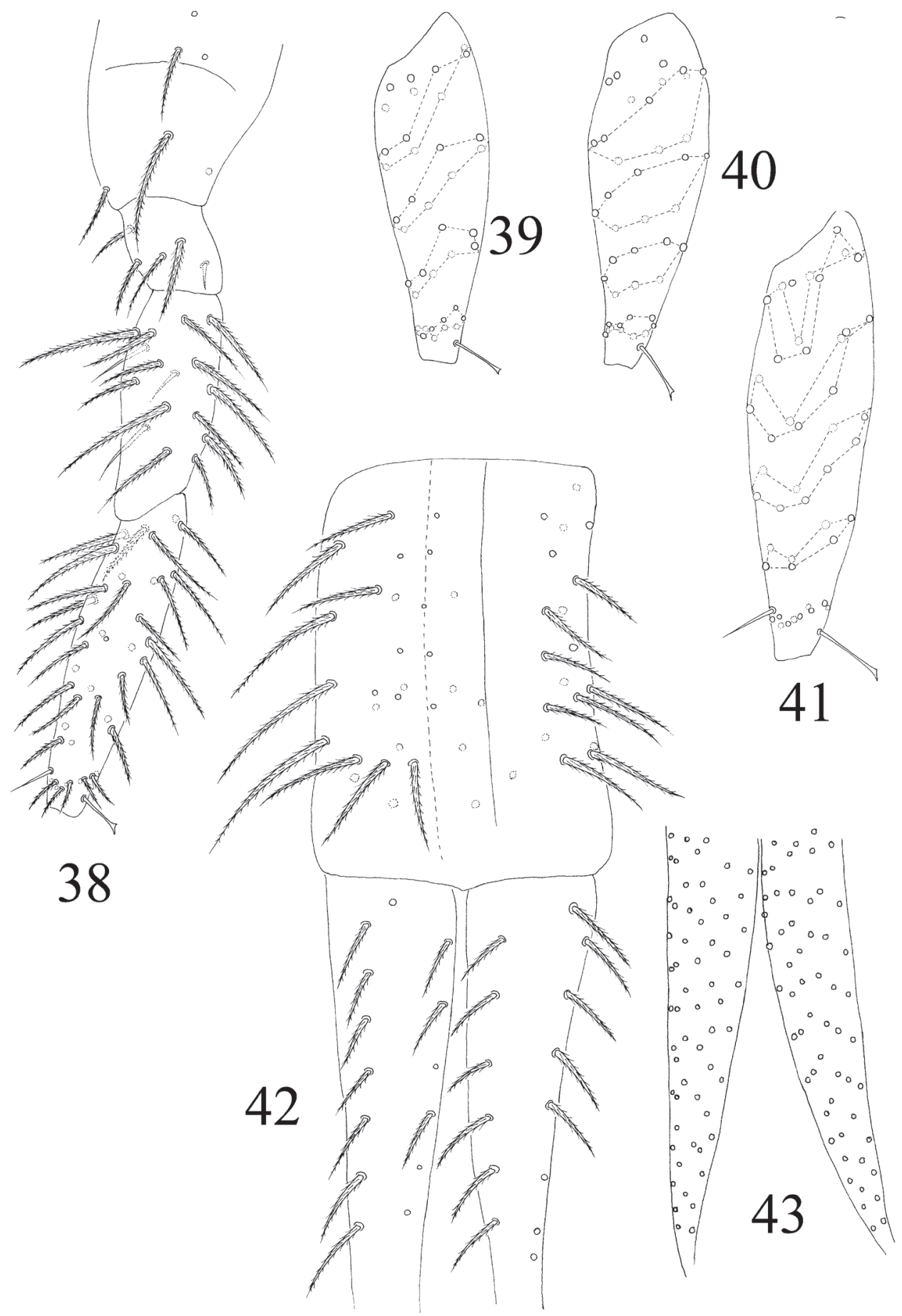

Figures 38-43. The first instar larval $H$. jordanai sp. n. 38 hind leg 39 Tita I 40 Tita II 4 I Tita III $\mathbf{4 2}$ manubrium and dorsal dens $\mathbf{4 3}$ ventral dens. 

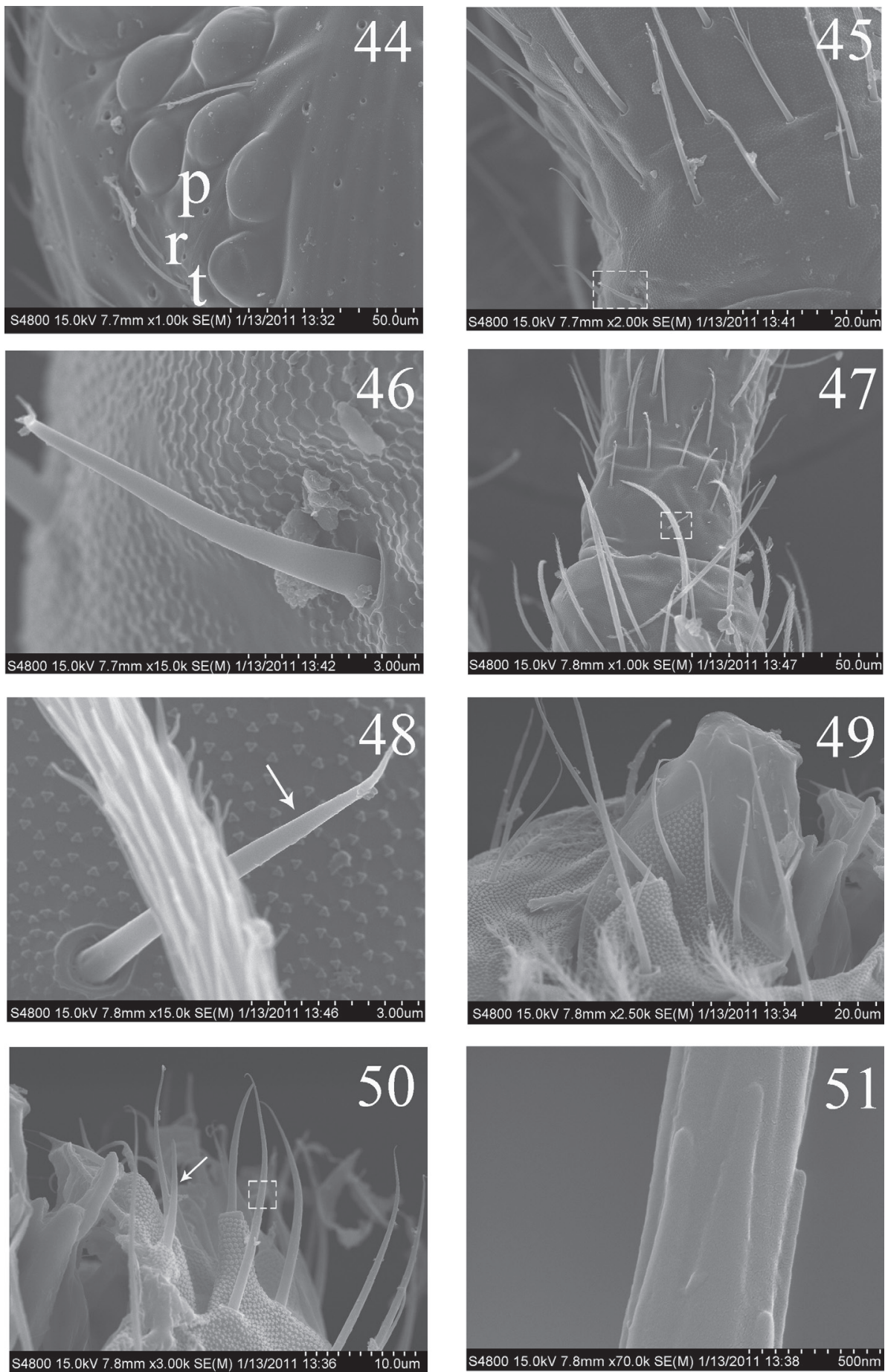

Figures 44-5I. SEM photos of adult $H$. jordanai sp. n. 44 eye patches $\mathbf{4 5}$ base of Ant. I, dorsal side $\mathbf{4 6}$ spiny setae on base of Ant. I, dorsal side $\mathbf{4 7}$ joint of Ant. I and Ant. II $\mathbf{4 8}$ spiny seta on base of Ant. II 49 maxillary outer lobe $\mathbf{5 0}$ labial palp $\mathbf{5}$ I micro-architecture of proximal setae. 

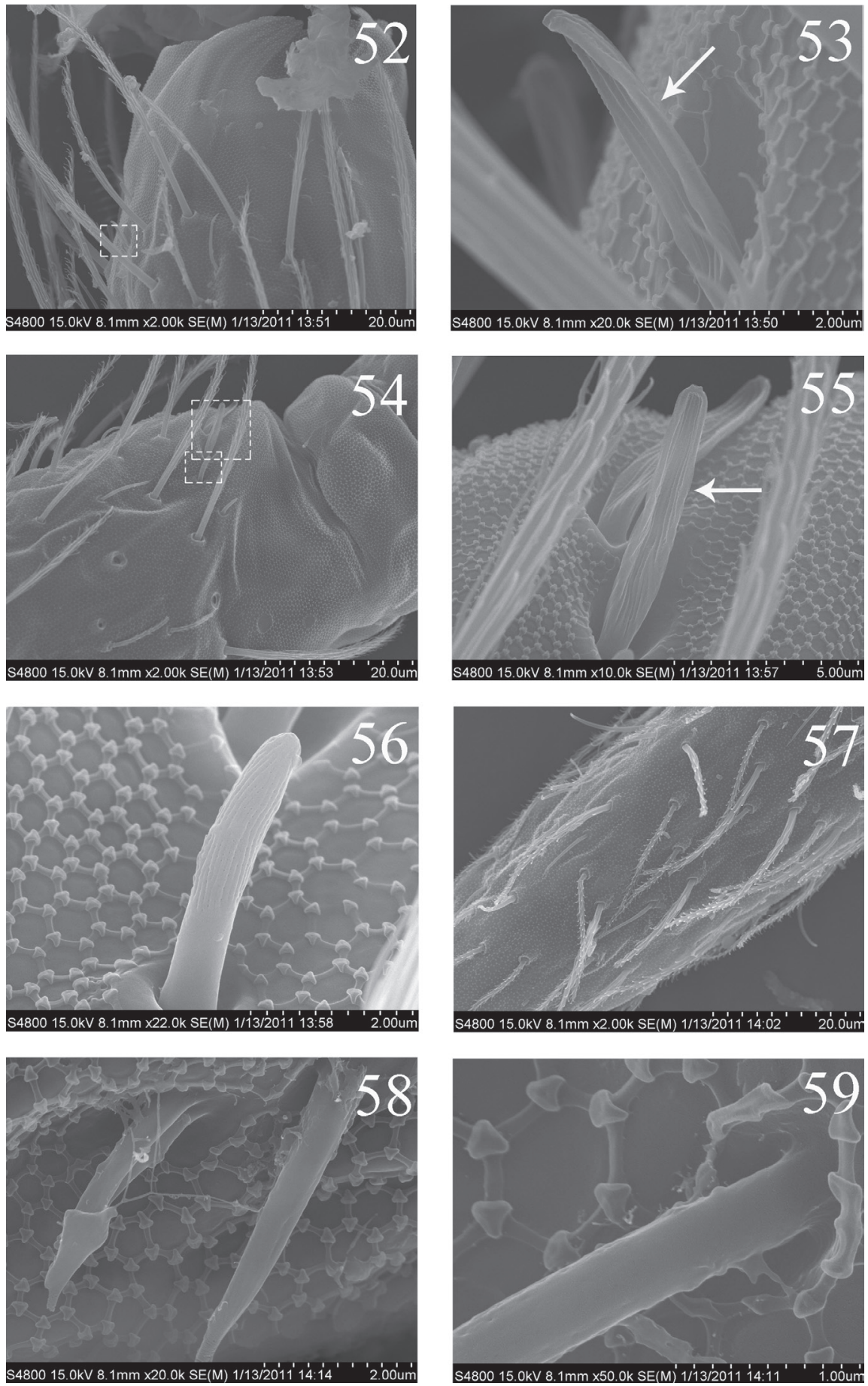

Figures 52-59. SEM photos of adult $H$. jordanai sp. n. 52 distal part of Ant. II $\mathbf{5 3} \mathrm{s}$ on distal part of Ant. II 54 Ant. III organ 55 internal two s of Ant. III organ $\mathbf{5 6}$ external s of Ant. III organ $\mathbf{5 7}$ Ant. IV 58-59 s on Ant. IV. 

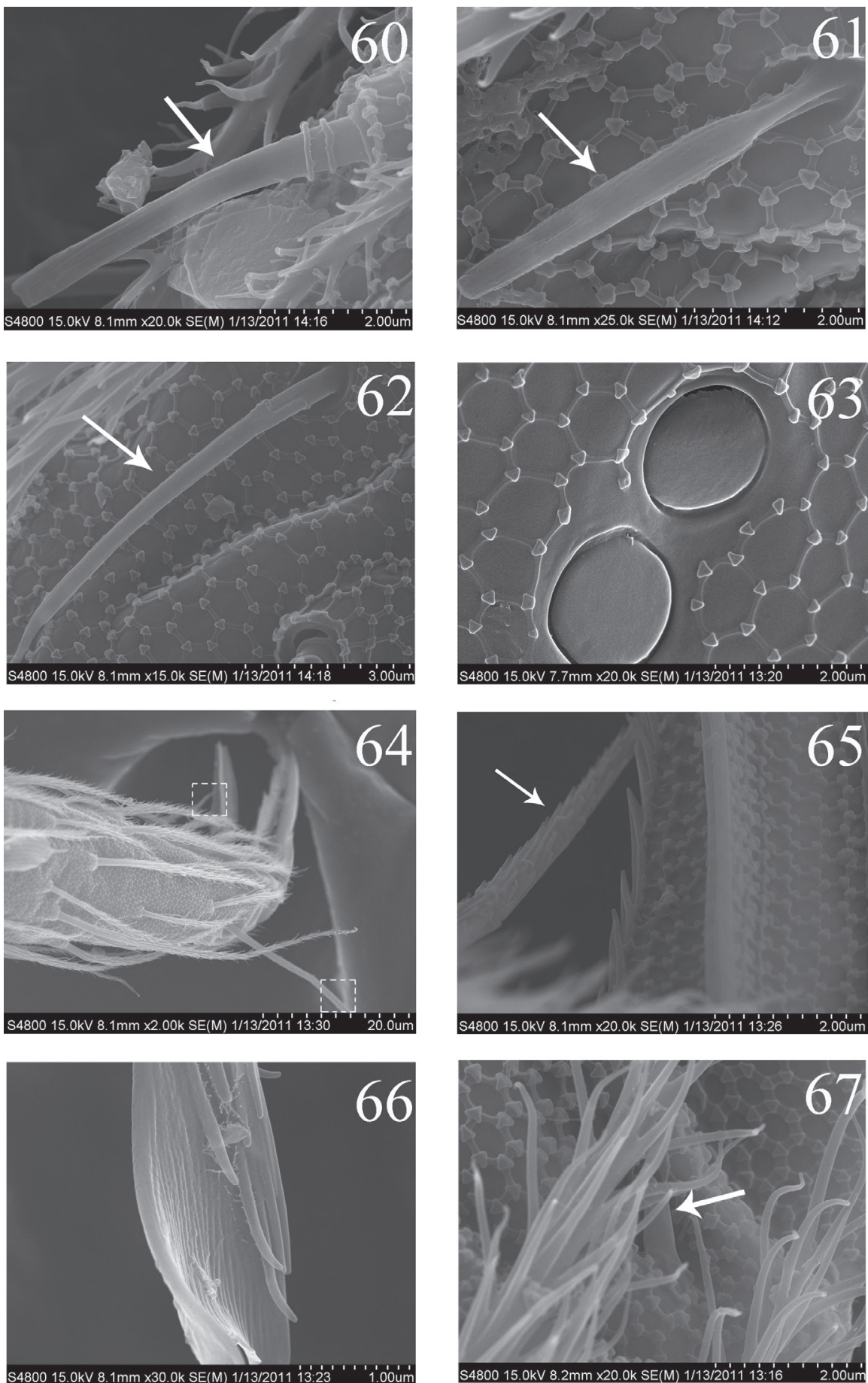

Figures 60-67. SEM photos of adult $H$. jordanai sp. n. 60-62 s of Ant. IV 63 pseudopores of coxa 64 Tita and claw of hind leg 65 supraempodial seta and outer edge of unguiculus 66 distal part of tenent hair 67 spiny seta on pretarsus. 

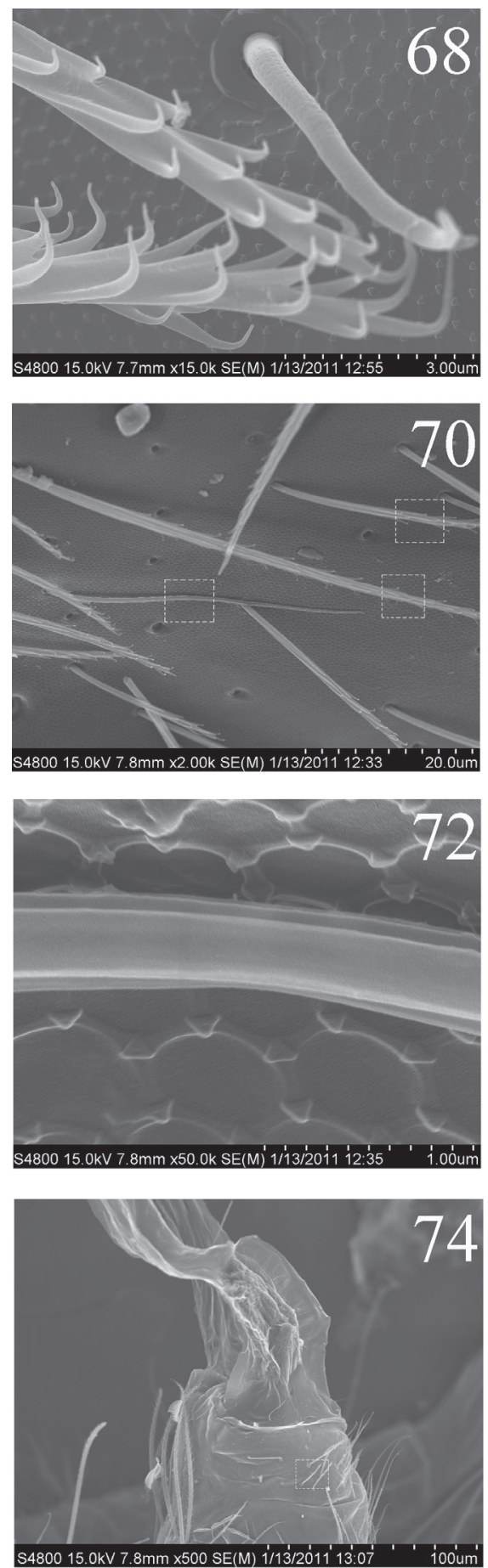
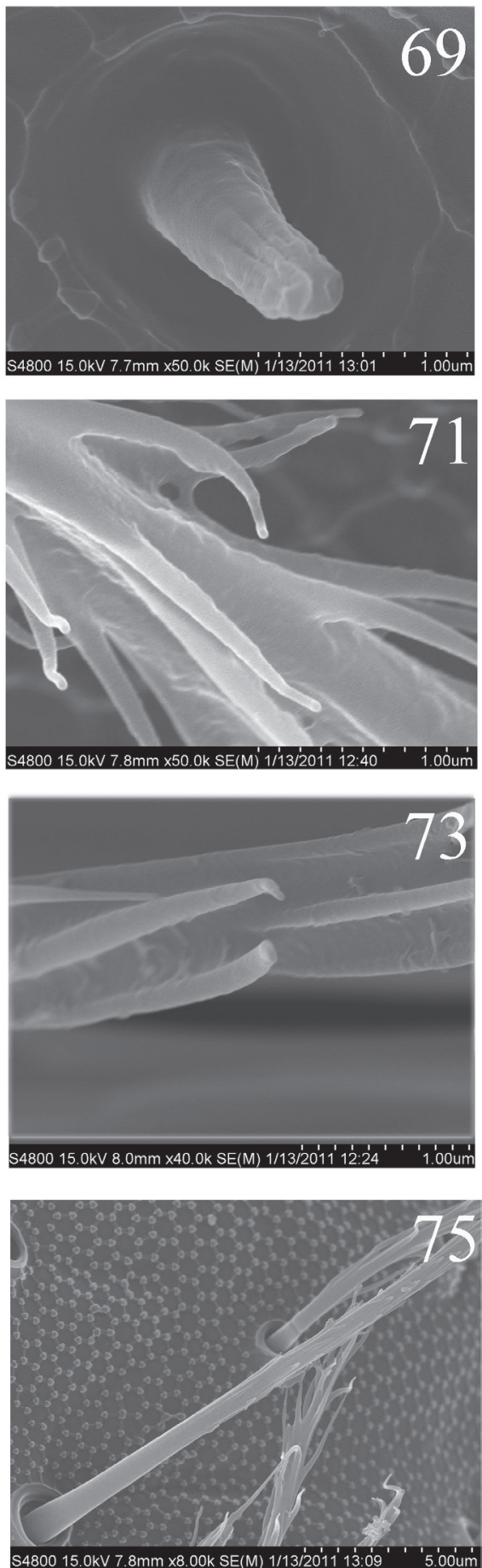

Figures 68-75. SEM photos of adult $H$. jordanai sp. n. 68 lateral s-chaetae on Abd. I 69 lateral ms on Abd. I 70 three types of setae on Abd. IV 7 I bothriotrichum on Abd. IV 72 elongate s-chaetae on Abd. IV $\mathbf{7 3}$ ciliate seta on Abd. IV $\mathbf{7 4}$ distal part of ventral tube $\mathbf{7 5}$ two types of setae on lateral flap of ventral tube. 

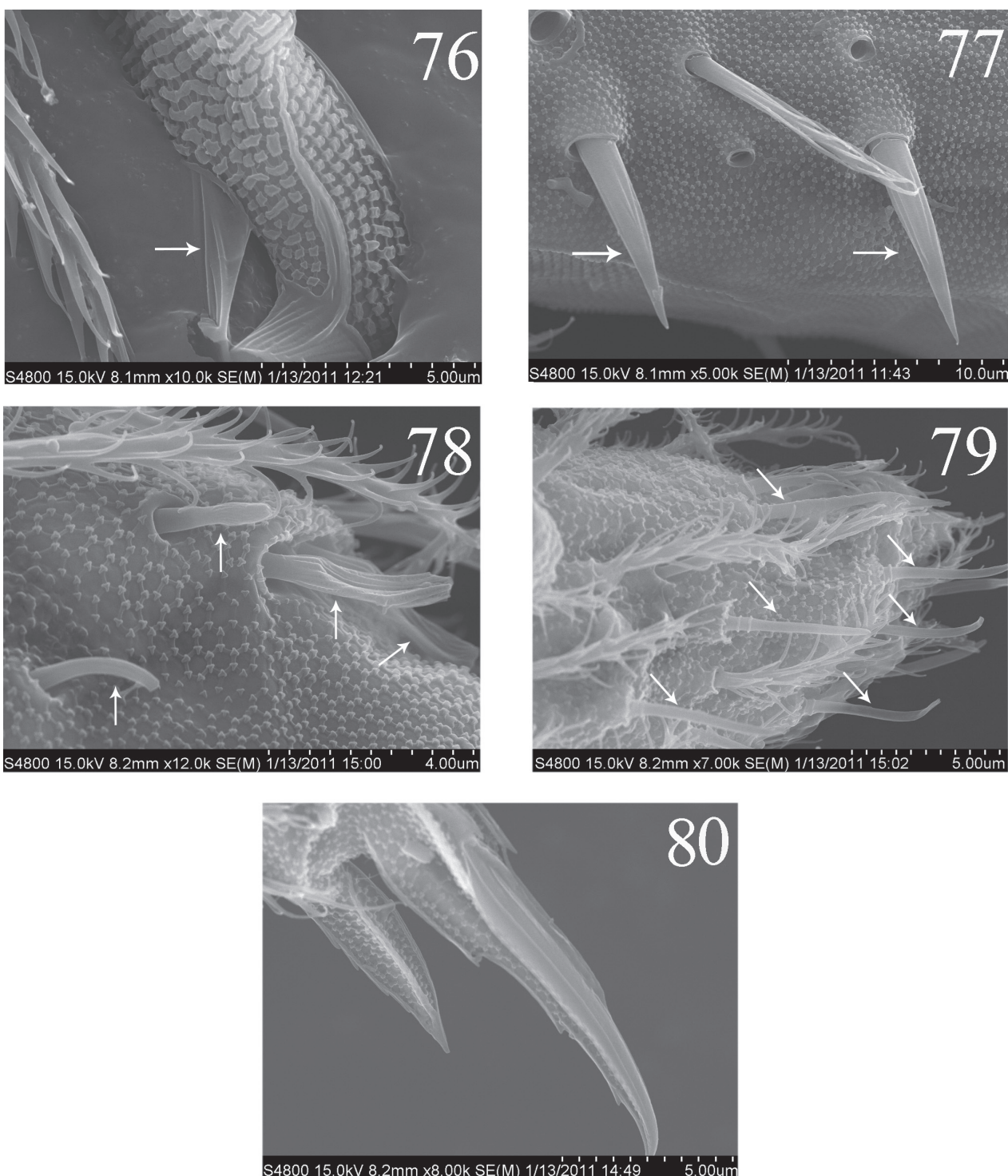

Figures 76-80. SEM photos of $H$. jordanai sp. n. 76-77 adult $\mathbf{7 6}$ partial mucro $\mathbf{7 7}$ dental spines 78-80 the first instar larva $\mathbf{7 8}$ Ant. III organ $\mathbf{7 9}$ distal part of Ant. IV 80 distal part of hind leg.

Mucro bidentate with subapical tooth obviously larger than apical one; basal spine absent. Tenaculum with $4+4$ teeth but without setae on corpus (Fig. 37).

Ecology. In the leaf litter of Cunninghamia lanceolata (Lambert) and Dicranopteris dichotoma (Thunberg).

Remarks. The new species is characterized by weak pigment on dorsal body, long antennae subequal to body in length, labial basal seta $\mathrm{L}_{1}$ ciliate, absence of setae $\mathrm{m} 2 \mathrm{i} 2$ on Th. II, a1 on Abd. I, a2 on Abd. III, A4-5 on Abd. IV, and dental basal seta pi spiny and shorter than bs. It is similar to Homidia unichaeta Pan, Shi \& Zhang, 2010 
Table I. Main differences between H. jordanai sp. n. and two similar species.

\begin{tabular}{l|l|l|l}
\hline Characters & $H . j$ & $H . u$ & $H . t$ \\
\hline Pigment on dorsal tergite & - & - & slight \\
\hline Antenna length as long as cephalic diagonal & $3.9-4.6$ & $1.5-2.5$ & about 3.5 times \\
\hline Seta L on labial base & ciliate & smooth & smooth \\
\hline Lateral prosess of labial papilla E & reach apex & not reach & $?$ \\
\hline Seta m2i2 on Th. II & - & + & - \\
\hline Seta a1 on Abd. I & - & + & + \\
\hline Seta a2 on Abd. III & - & + & + \\
\hline Mac on posterior Abd. IV & 2 (rarely 3) & 1 & 2 \\
\hline "Eyebrow" setae on anterior Abd. IV & $6-9$ & $3-8$ (usually 5-7) & $10-12$ \\
\hline Dental spines & $20-40$ & $19-23$ & $39-54$ \\
\hline Comparison of dental basal seta in length & bs $>$ pi & pi $>$ bs & bs $>$ pi \\
\hline Type locality (China) & Zhejiang & Zhejiang & Tibet \\
\hline
\end{tabular}

H. j: H. jordanai sp. n.; H. u: H. unichaeta; H. t. H. tibetensis; -: absent; +: present;?: the character unclear.

Table 2. Differences between the first instar larvae and adults (apart from chaetotaxy of body tergites).

\begin{tabular}{l|l|l}
\hline Characters & First instar larvae & Adults \\
\hline Ground colour & pale white & pale yellow \\
\hline Cephalic chaetotaxy & 3 An, 5S & 4An, 7S \\
\hline Spiny setae on basal Ant. I & 1 & 2 dorsal, 4 ventral \\
\hline S on distal Ant. II & - & 4 \\
\hline Proximal setae of labium & 3 & 5 \\
\hline Seta R on labial base & - & + \\
\hline Coxal macrochaetal formula & $2 / 3 / 5$ & $3 / 4+1,3 / 4+2$ \\
\hline Setae on trochanteral organ of hind leg & 1 & $36-64$ \\
\hline Inner tibiotarsal setae & strongly ciliate & thicker and slightly serrate \\
\hline Setae on anterior face of ventral tube & - & $4+4$ mac and numerous mic \\
\hline Setae on lateral flap of ventral tube & 2 smooth & $6-7$ smooth and 10-24 ciliate \\
\hline Setae on posterior face of ventral tube & 2 smooth & 5 or 6 smooth and numerous ciliate \\
\hline Seta on tenaculum & - & 1 \\
\hline "Eyebrow" setae on Abd. IV & - & $6-9$ \\
\hline Setae on manubrium & $24+24$ & more than $24+24$ \\
\hline Pseudopores on manubrial plaque & - & 3 \\
\hline Ciliate setae on manubrial plaque & - & $7-8$ \\
\hline Basal setae (bs and bs ) on dens & - & + \\
\hline The shape of proximal-inner seta (pi) & normal & spiny \\
\hline Dental spines & - & $20-40$ \\
\hline Basal spine of mucro & - & + \\
\hline Genital plate & - & papillate \\
\hline
\end{tabular}

-: absent; +: present. 
and Homidia tibetensis Chen \& Zhong, 1998 in colour pattern, cephalic chaetotaxy, labrum, coxal formula, chaetotaxy of Abd. II and claw. However, it can be distinguished from them by the length of antennae, labial setal formula, chaetotaxy on Th. II, Abd. I, Abd. III-IV and seta pi on basal dens. Additional differences are listed in Table 1.

Differences between the first instar larvae and adults. Some characters are principally same in the first instar larvae and adults: ommatidia, interocular setae, Ant. III organ, apical bulb on Ant. IV, labrum and labral papillae, labial palp, maxillary outer lobe, claw, bothriotricha and s-chaetoxic pattern on terga.

Characters that develop after the first instar: s on distal part of Ant. II, smooth setae on base of Ant. II, labial seta R, smooth spiny setae on trochanteral organ, mac on anterior face of ventral tube, seta on corpus of tenaculum, pseudopores on manubral plaque, basal spine on mucro and genital plate.

Chaetotaxy become more complicated during postembryonal development, detailed differences between the first instar larvae and adults (apart from chaetotaxy of body tergites) are listed in Table 2. Tergal chaetotaxy of adults becomes much more complicated than that of primary chaetotaxy. In addition to numerous secondary common mic and mac on terga, some primary mic are transformed into mac: $\mathrm{m} 2, \mathrm{~m} 5$ and p4 on Th. II, a1, m5, p5 and p6 on Th. III, a2, a3 and a5 on Abd. I, a2 and a3 on Abd. II, am 6 and p6 on Abd. III, A6 and B4 on Abd. IV (homology of lateral setae difficult to determine).

\section{Discussion}

Taxonomical significance of the first instar larvae and the position of the genus Homidia

The adult chaetotaxy of Entomobryidae exhibits marked differences among genera or species. Szeptycki (1972) found that the primary chaetotaxy of Entomobryomorpha was almost identical in number and position. Later, he (1979) studied the ontogeny of tergal chaetotaxy of the representative Entomobryidae genera and its preliminary phylogenetic significance at higher level of hierarchy with four subfamilies included in Entomobryidae. Subsequent authors (Rusek 2002; Deharveng 2004) emphasized again the systematic significance of the first instar larval chaetotaxy at higher level.

We compare the primary dorsal chaetotaxy of the body among $H$. jordanai sp. n. and another five species of family Entomobryidae. The morphology of some primary homologous setae under Szeptycki's nomenclature exhibits stable differentiation (mac, mic or absent) at the first instar (Table 3), though number and position of them are apparently similar. Among six species, the primary tergal chaetotaxy of $H$. jordanai sp. n. is closest to Entomobryoides myrmecophila, almost identical in number and arrangement except s-chaetae on Abd. IV. Integrating its adult features, such as 
Table 3. Morphological differences of the first instar larvae among six Entomobyridae species.

\begin{tabular}{|c|c|c|c|c|c|c|c|}
\hline tergite & seta & $H . j$ & O.f & H. $n$ & E. $m$ & P. $a$ & S.d \\
\hline \multirow[t]{3}{*}{ Th. II } & $\mathrm{m} 1$ & $\mathrm{mac}$ & $\mathrm{mac}$ & mic & $\mathrm{mac}$ & $\mathrm{mac}$ & $\mathrm{mac}$ \\
\hline & $\mathrm{m} 2$ & mic & mac & mic & mic & scale & $\mathrm{mac}$ \\
\hline & $\mathrm{p} 5$ & $\mathrm{mac}$ & mac & mic & mic & $\mathrm{mac}$ & $\mathrm{mac}$ \\
\hline \multirow[t]{5}{*}{ Th. III } & $\mathrm{a} 2$ & $\mathrm{mac}$ & mic & mic & mic & mic & mac \\
\hline & a3 & mac & mic & mic & mic & mic & mic \\
\hline & $\mathrm{a} 4$ & $\mathrm{mac}$ & $\mathrm{mac}$ & mic & mic & mac & $\mathrm{mac}$ \\
\hline & $\mathrm{m} 1$ & mic & mic & mic & mic & - & mic \\
\hline & $\mathrm{m} 2$ & - & - & - & - & $\mathrm{mac}$ & - \\
\hline \multirow[t]{3}{*}{ Abd. I } & $\mathrm{m} 2$ & $\mathrm{mac}$ & $\mathrm{mac}$ & $\mathrm{mac}$ & mic & mac & $\mathrm{mac}$ \\
\hline & $\mathrm{m} 4$ & $\mathrm{mac}$ & $\mathrm{mac}$ & mic & mac & $\mathrm{mac}$ & $\mathrm{mac}$ \\
\hline & $\mathrm{m} 6$ & mic & mic & mic & $\mathrm{mic}$ & mic & mic \\
\hline \multirow[t]{2}{*}{ Abd. II } & $\mathrm{a} 2$ & mic & mac & mic & mic & $\mathrm{mac}$ & $\mathrm{mac}$ \\
\hline & $\mathrm{m} 5$ & $\mathrm{mac}$ & $\mathrm{mac}$ & mic & $\mathrm{mac}$ & mac & $\mathrm{mac}$ \\
\hline \multirow[t]{2}{*}{ Abd. III } & pm6 & $\mathrm{mac}$ & mac & mac & mac & $\mathrm{mac}$ & $\mathrm{mac}$ \\
\hline & p6 & mic & mic & mic & $\mathrm{mac}$ & mic & $\mathrm{mac}$ \\
\hline \multirow[t]{7}{*}{ Abd. IV } & A4 & mic & - & - & $\mathrm{mic}$ & - & - \\
\hline & A5 & - & - & - & - & mic & mic \\
\hline & B4 & mic & - & - & mic & mic & $\mathrm{mac}$ \\
\hline & B5 & mac & mic & mic & mac & mac & $\mathrm{mac}$ \\
\hline & B6 & mic & mic & - & $\mathrm{mic}$ & mic & mic \\
\hline & E2 & - & $\mathrm{mac}$ & mic & - & mic & mic \\
\hline & E3 & mac & - & - & mac & mic & $\mathrm{mac}$ \\
\hline
\end{tabular}

H. j: Homidia jordanai sp. n.; O. f. Orchesella flavescens (Bourlet, 1839); H. n: Heteromurus nitidus (Templeton, R in Templeton, R \& Westwood, J. O, 1836); E. m: Entomobryoides myrmecophila (Reuter, 1884); P. a: Pseudosinella alba (Packard, 1873); S. d: Seira dowlingi (Wray, 1953); -: absent.

elongated Abd. IV, four antennal segments and absence of scales, the genus Homidia apparently belongs Entomobryini sensu (Soto-Adames et al. 2008). It differs from Orchesellinae in 4 antennal segments (5-6 in the latter), elongated Abd. IV, as well as more primary setae on Abd. IV. It also can be distinguished from Seira and Lepidocyrid species by presence of $\mathrm{p} 4$ and absence of $\mathrm{p} 3$ (reverse in the latter) on Abd. III, and absence of E2 on Abd. IV. Among genera of Entomobryini (Entomobrya, Drepanura, Sinella, Coecobrya), adults of Homidia is easily separated from others in presence of dental spines, "eyebrow" on Abd. IV, and larger subapical mucronal tooth; as for first instar larvae (no dental spines), we also separate it from others by below characters: smooth labial seta E, abundant elongated s-chaetae on Abd. IV (much more than primary setae), and longer distance between area $\mathrm{aM}$ and $\mathrm{pM}$ on Abd. IV. The origin of peculiar "eyebrow" on anterior part of Abd. IV of adults couldn't be traced by primary chaetotaxy; postembryonic development may provide key evidences to homology of enigmatic "eyebrow". 
It is still a long way to achieve the correct homology of setae in Entomobryidae. Further exploration at species level could be studied by thorough survey of more species and more characters of first instar, although first instar larvae are usually more difficult to collect than adults and subadults.

\section{Morphology of the "smooth" setae under SEM}

Spiny setae on antennae are smooth under LM and SEM. S and ms on dorsal body smooth under LM, but not smooth under SEM (Figs 53-62, 68, 69, 72). Guard setae on labial palp, proximal setae, "smooth" setae on ventral tube, tenent hair and supraempodial seta on Tita III are also smooth under LM, but weakly ciliate under SEM (Figs 50, 51, 64-66, 74, 75). We have to carefully describe "smooth" setae in future (Chen and Christiansen 1993). Descriptions of some details (e. g. setal surface) based on LM are incomplete, and may bring confusion. SEM observation could provide fine details as a better supplementary tool for species diagnosis.

\section{Acknowledgements}

We thank to Dr Frans Janssens (Belgium) and Prof. Christiansen K (USA) who provided some necessary literature for us. The present study was supported by The Zhejiang Provincial Natural Science Foundation of China (Y3100278 and Y3100149) and The Zhejiang Provincial Department of Educational Foundation of China (Y200700872).

\section{References}

Chen JX, Christiansen K (1993) The genus Sinella with special reference to Sinella s.s. (Collembola: Entomobryidae) of China. Oriental Insects 27: 1-54.

Chen JX, Li LR (1997) A new species of the genus Homidia (Collembola: Entomobryidae) from Jiangxi province of China. Entomotaxonomia 19: 79-82.

Chen JX, Zhong WY (1998) A new species of the genus Homidia (Collembola: Entomobryidae) from Xizang Autonomous Region, China. Entomologia Sinica 5: 50-54.

Christiansen K (1992) Springtails. The Kansas School Naturalist 39: 1-15.

Deharveng L (2004) Recent advances in Collembola systematics. Pedobiologia 48: 415-433. doi: $10.1016 /$ j.pedobi.2004.08.001

Fjellberg A (1998) The labial palp in Collembola. Zoologischer Anzeiger 237: 309-330.

Gisin H (1964) Collemboles d'Europe VII. Revue Suisse de Zoologie 71: 649-678.

Jordana R, Baquero E (2005) A proposal of characters for taxonomic identification of Entomobrya species (Collembola, Entomobryomorpha), with description of a new species. Abhandlungen und Berichte des Naturkundemuseums Goerlitz 76: 117-134. 
Mari-Mutt JA (1979) A revision of the genus Dicranocentrus Schött (Insecta: Collembola: Entomobryidae). Agricultural Experiment Station University Puerto Rico Bulletin 259: 1-79.

Mari-Mutt JA (1986) Puerto Rican species of Lepidocyrtus and Pseudosinella (Collembola: Entomobryidae). Caribbean Journal of Science 22: 1-48.

Pan ZX, Shi SD, Zhang F (2010) A new species of genus Homidia (Collembola: Entomobryidae) from Zhejiang Province, China. Entomotaxonomia 32: 241-247.

Rusek J (2002) Taxonomy of Collembola at the beginning of the new millennium. Pedobiologia 46: 215-224. doi: 10.1078/0031-4056-00128

Soto-Adames FN (2008) Postembryonic development of the dorsal chaetotaxy in Seira dowlingi (Collembola, Entomobryidae); with an analysis of the diagnostic and phylogenetic significance of primary chaetotaxy in Seira. Zootaxa 1683: 1-31.

Soto-Adames FN, Barra JA, Christiansen KA, Jordana R (2008) Suprageneric Classification of Collembola Entomobryomorpha. Annals of the Entomoligical Society of America 101: 501-513. doi: 10.1603/0013-8746(2008)101[501:SCOCE]2.0.CO;2

Szeptycki A (1973) North Korean Collembola. I. The genus Homidia Börner, 1906 (Entomobryidae). Acta Zoologica Cracoviensia 31: 23-39.

Szeptycki A (1979) Morpho-systematic studies on Collembola. IV. Chaetotaxy of the Entomobryidae and its phylogenetical significance. Polska Akademia Nauk, Kraków, Poland, 216 pp. 